\title{
Significance of congenital coronary artery fistulas
}

Submitted: 15 June 2016; Accepted: 11 July 2016; Published online: 16 July 2016

Congenital coronary artery fistulas (CAFs) are anomalous terminations of coronary arteries in cardiac chambers or great arteries created by bypassing the myocardial capillary bed. CAFs are the second most common coronary anomaly after coronary artery origin anomalies [1]. CAFs are mostly asymptomatic. However, CAFs may cause myocardial ischemia, angina pectoris, congestive heart failure, arrhythmias and infective endocarditis [2]. Previously, we presented a case of multiple coronarycameral fistulas causing stable angina pectoris [3]. A 65-year-old male patient referred to our outpatient clinic with chest pain and exertional dyspnea. The patient was suffering from retrosternal exertional chest pain for six months which relieved with rest. Previous medical history revealed hypertension and diabetes mellitus. Electrocardiogram showed inferolateral $\mathrm{T}$ wave inversion (Figure $\mathrm{A}$ ) and echocardiography revealed normal left ventricular systolic function without left ventricular hypertrophy or cardiomyopathy. Coronary angiography was performed with an early diagnosis of coronary artery disease. There was not any significant coronary stenosis, however in late phase of contrast injection to the left coronary arteries, left ventricle filling and opacification was seen through extensive multiple

microfistulas (Figures B and C). Because of extensive spreading of microfistulas through left ventricle myocardium, the patient was not an appropriate candidate for surgical or transcatheter repair and regular follow-up with medical therapy was planned. Symptomatic relief was provided by medical therapy with acetylsalicylic acid, ramipril, amlodipine, metoprolol and atorvastatin.

CAFs mostly originate from the right coronary artery system and drain through the right heart chambers. A total of $25 \%$ CAFs originate from the left coronary artery system [4]. Pulmonary arterial fistulization is $17 \%$ in single CAF, whereas this rate increases to $56 \%$ in bilateral CAF [5]. Being mostly asymptomatic, CAFs is usually suspected by hearing a murmur during a physical examination and is diagnosed in further evaluation or incidentally in diagnostic CAG. Hemodynamically significant fistulas can cause symptoms such as angina or dyspnoea.
Umut Kocabas*

Department of Cardiology, Soma State Hospital, Soma, Manisa, Turkey *Author for correspondence: Tel.: +905079974999 umutkocabas@hotmail.com
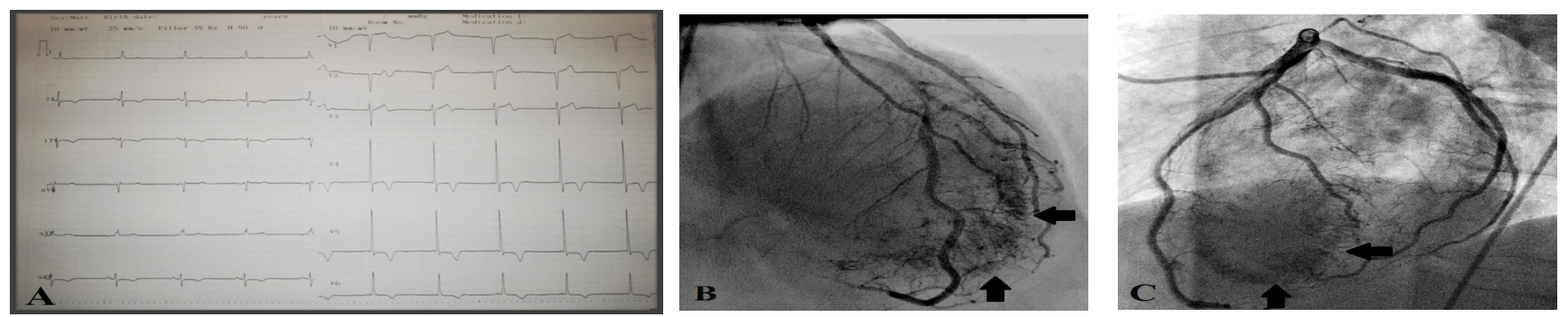

Figure 1: A: The twelve-lead electrocardiogram showed left ventricular hypertrophy and inferolateral T wave inversion.

Figure B: In Late phase of contrast injection to the left coronary arteries.

Figure C: Left ventricle Opacification was seen through extensive multiple corono-cameral microfistulas. 
Angina is usually associated with coronary steal phenomenon. In this phenomenon, coronary blood flow is shifted to the territory of the fistula because of low blood pressure in the diastole. Consequently, sufficient blood supply cannot be delivered and ischemia occurs in the distal territory of the fistulabearing artery. Aging increases the risk of dilatation and/or aneurysm formation of the fistula, thereby increasing the risk of complications [6]. The incidence of symptoms or complications in individuals $<20$ years is $19 \%$, and increases to $55 \%$ years after that age [7]. Asymptomatic and small-diameter CAFs to must be followed medically. The main indications for surgical or interventional treatment of CAFs are hemodynamically important fistulas, symptomatic status, myocardial ischemia, heart failure, arrhythmias, or infective endocarditis [2]. The main purpose of treatment is closure of the fistula without damaging coronary blood

\section{References}

1. Levin DC, Fellows KE, Abrams HL. Hemodynamically significant primary anomalies of the coronary arteries. Angiographic aspects. Circulation. 58, 25-34 (1978).

2. Gowda RM, Vasavada BC, Khan IA. Coronary artery fistulas: clinical and therapeutic considerations. Int. J. Cardiol. 107: 7-10 (2006).

3. Kocabas U, Kaya E, Akin M. Coronary injection ventriculography: Multiple coronary-cameral fistulas as a rare cause of stable angina pectoris. Int. J. Cardiol. Acad. 2, 68-70 (2016).

4. Fernandes ED, Kadivar H, Hallman GL, et al. Congenital malformations of the coronary arteries: the Texas Heart Institute experience. Ann. Thorac. Surg. 54, 732-740 (1992). flow. Surgical therapy or transcatheter coil embolization, which is currently in wider use, can be performed. The efficacy of transcatheter coil embolization is similar to surgical therapy in terms of morbidity and mortality rates. However, this method is superior to surgical therapy according to patient comfort and duration of hospitalization. Surgery is performed in patients who cannot undergo transcatheter coil embolization [8]. In our case, due to multiple small calibre CCFs, neither surgical nor transcatheter therapy were found appropriate.

In conclusion, hemodynamically significant CAFs may cause myocardial ischemia, angina pectoris, arrhythmias, infective endocarditis and heart failure. Surgical or interventional treatment can be performed in this group of patients. However, multiple small calibre CCFs are not suitable for surgical or interventional treatment strategies.

5. Bauer EP, Piepho A, Klövekorn WP. Coronary arteriovenous fistula: surgical correction of a rare form. Thorac. Cardiovasc. Surg. 42, 237-239 (1994).

6. Sunder KR, Balakrishnan KG, Tharakan JA, et al. Coronary artery fistula in children and adults: a review of 25 cases with long-term observations. Int. J. Cardiol. 58: 47-53 (1997).

7. Liberthson RR, Sargar K, Berkoben JB, Weintraub RM, Levine FH. Congenital coronary arteriovenous fistula. Report of 13 patients, review of the literature and delineation of management. Circulation. 59, 849-854 (1979).

8. Armsby LR, Keane JF, Sherwood MC, et al. Management of coronary artery fistulae. Patient selection and results of transcatheter closure. J. Am. Coll. Cardiol. 39, 1026-1032 (2002). 